\title{
Mechanisms of iron sensing and regulation in the yeast Saccharomyces cerevisiae
}

María Teresa Martínez-Pastor ${ }^{*}$, Ana Perea-García ${ }^{2}$ and Sergi Puige*

${ }^{1}$ Departamento de Bioquímica y Biología Molecular, Universitat de València, Burjassot, Valencia, Spain.

2Departamento de Biotecnología, Instituto de Agroquímica y Tecnología de Alimentos (IATA), Consejo Superior de Investigaciones Científicas (CSIC), Paterna, Valencia, Spain.

*Corresponding authors: E-mail: maria.teresa.martinez@uv.es; spuig@iata.csic.es

Running title: Iron sensing in yeast

Keywords: iron homeostasis, iron deficiency, Fe-S cluster synthesis, yeast, Saccharomyces cerevisiae, Aft1, Yap5 


\section{Abstract}

Iron is a redox active element that functions as an essential cofactor in multiple metabolic pathways, including respiration, DNA synthesis and translation. While indispensable for eukaryotic life, excess iron can lead to oxidative damage of macromolecules. Therefore, living organisms have developed sophisticated strategies to optimally regulate iron acquisition, storage and utilization in response to fluctuations in environmental iron bioavailability. In the yeast Saccharomyces cerevisiae, transcription factors Aft1/Aft2 and Yap5 regulate iron metabolism in response to low and high iron levels, respectively. In addition to producing and assembling iron cofactors, mitochondrial iron-sulfur (Fe/S) cluster biogenesis has emerged as a central player in iron sensing. A mitochondrial signal derived from Fe/S synthesis is exported and converted into an Fe/S cluster that interacts directly with Aft1/Aft2 and Yap5 proteins to regulate their transcriptional function. Various conserved proteins, such as $\mathrm{ABC}$ mitochondrial transporter Atm1 and, for Aft1/Aft2, monothiol glutaredoxins Grx3 and Grx4 are implicated in this iron-signaling pathway. The analysis of a wide range of $S$. cerevisiae strains of different geographical origins and sources has shown that yeast strains adapted to high iron display growth defects under iron-deficient conditions, and highlighted connections that exist in the response to both opposite conditions. Changes in iron accumulation and gene expression profiles suggest differences in the regulation of iron homeostasis genes. 


\section{Introduction}

Iron is an essential micronutrient for all eukaryotic organisms because it functions as an essential redox cofactor in multiple processes, including cellular respiration, lipid biosynthesis, translation and amino acid biogenesis, DNA replication and repair, oxygen transport, photosynthesis or nitrogen fixation. However, iron redox properties can be deleterious when iron is present in excess because it can damage cells at the level of nucleic acids, proteins and lipids. For this reason, living organisms have developed complex strategies to modulate iron metabolism. The budding yeast Saccharomyces cerevisiae has been used as a model eukaryotic organism to study many aspects of iron homeostasis, including iron transport, distribution and regulation, and to decipher the molecular bases of multiple iron-related diseases (reviewed in Philpott et al. 2012; Sanvisens and Puig 2011).

Yeast cells respond to alterations in iron bioavailability by modifying the expression of multiple genes. Two transcription factors, Aft1 and Aft2 (Aft1/2), coordinate the activation of genes upon iron scarcity, whereas Yap5 regulates specific genes in response to excess iron (Philpott et al. 2012; Pimentel et al. 2012; Sanvisens and Puig 2011). The molecular mechanisms that yeast cells utilize to perceive and respond to iron fluctuations have only recently been addressed. These studies have uncovered an unanticipated role for the $\mathrm{Fe} / \mathrm{S}$ cluster biosynthesis pathway in the regulation of iron homeostasis (for extended reviews, see Lill et al. 2014; Muhlenhoff et al. 2015; Outten and Albetel 2013). Mitochondrial $\mathrm{Fe} / \mathrm{S}$ cluster assembly is an essential process conserved from yeast to humans that is required for the maturation of all $\mathrm{Fe} / \mathrm{S}$ cluster-containing proteins. Briefly, inner membrane carriers Mrs3 and Mrs4 import iron into 
mitochondria. Then the cysteine desulfurase complex Nfs1-Isd11 and frataxin facilitate the assembly of a [2Fe-2S] cluster on scaffold protein Isu1. The cluster is then transferred to apoproteins with the help of a dedicated chaperone system and monothiol glutaredoxin Grx5. The components that participate in these initial stages are the so-called "core Fe/S cluster proteins" because they are essential for the maturation of all $\mathrm{Fe} / \mathrm{S}$ proteins. Specialized $\mathrm{Fe} / \mathrm{S}$ components assist in the

generation and insertion of [4Fe-4S] clusters into specific target apoproteins. A product of the core $\mathrm{Fe} / \mathrm{S}$ cluster machinery is exported from mitochondria by conserved $\mathrm{ABC}$ transporter Atm 1 within the mitochondrial inner membrane, and is then distributed to cytoplasmic and nuclear Fe/S cluster-containing proteins by the cytoplasmic Fe/S cluster assembly (CIA) machinery. In this minireview, we focus on recent findings about the mechanisms that $S$. cerevisiae utilizes to transmit cellular iron status, and place special emphasis on the effect that the mitochondrial Fe/S cluster synthesis pathway has on Aft1/Aft2 and Yap5 ironregulated transcription factors.

\section{Regulation of $A f t 1$ and $A f t 2$ in response to iron deficiency}

In response to iron deficiency, $S$. cerevisiae activates the expression of a group of $\sim 30$ genes collectively known as the iron regulon (Table 1). Iron regulon members include genes involved in: (i) high-affinity iron acquisition in the plasma membrane (metalloreductases, inorganic and siderophore iron transporters); (ii) intracellular iron transport (iron importer into mitochondria, iron exporters from the vacuole); (iii) iron recycling (heme oxygenase); and (iv) metabolic remodeling that aims to optimize iron utilization (Cth1 and Cth2 mRNA-binding proteins; biotin transporter). All iron regulon members contain at least one "iron regulatory 
element" (FeRE) within its promoter region, which is recognized by Aft1 and/or Aft2 transcription factors (Kaplan and Kaplan 2009). A recently solved crystal structure for Aft2 bound to DNA has demonstrated that this transcription factor recognizes the FeRE as a monomer through its WRKY-GCM1 $\mathrm{Zn}^{2+}$-binding domain (Poor et al. 2014). Under iron replete conditions, Aft1 (and probably Aft2) shuttles between the nucleus and the cytoplasm. Yet when iron becomes scarce, Aft1 accumulates in the nucleus, binding FeREs and activating transcription (Yamaguchi-Iwai et al. 2002). When iron concentrations are adequate, nuclear export receptor Msn5 interacts with Aft1 and Aft2 promoting export from the nucleus (Ueta et al. 2007). However, Aft1 export from the nucleus is not absolutely necessary to inhibit its transcriptional function because Aft1 activity is still abolished by iron in msn5 cells, in which Aft1 is constitutively present in the nucleus (Ueta et al. 2012). Further work with various Aft1 mutants has demonstrated that the iron-induced dissociation from DNA is a crucial event in Aft1 regulation by iron (Ueta et al. 2012). Aft1 plays the predominant iron regulatory function, whereas Aft2 is dedicated to activate the genes involved in intracellular iron transport (Blaiseau et al. 2001; Rutherford et al. 2001). Thus aft1 $\Delta$ cells display a severe growth defect under iron-limited conditions that is further exacerbated when the AFT2 gene is deleted (Blaiseau et al. 2001; Rutherford et al. 2001).

Various observations have initially suggested that yeast cells, specifically Aft1 and Aft2 transcription factors, perceive reductions in cytosolic iron concentrations ( $\left.\mathrm{Fe}_{\text {cyt }}\right)$ and respond accordingly by activating iron acquisition and mobilization genes. A delay in Aft1-mediated transcriptional activation is first observed when iron uptake increases or vacuolar iron storage diminishes (Chen et al. 2004). 
Second, yeast mutants defective in mitochondrial Fe/S cluster biogenesis or efflux accumulate high iron levels in mitochondria and activate the iron regulon (Chen et al. 2002; Hausmann et al. 2008). By using a modified iron-dependent enzyme within the sterol biosynthetic pathway to determine $\mathrm{Fe}_{c y t}$, Kaplan and colleagues conclude that decreasing $\mathrm{Fe} / \mathrm{S}$ cluster synthesis activates the iron regulon, even in iron replete cells (Chen et al. 2004). These results demonstrate that yeast mainly responds to mitochondrial $\mathrm{Fe} / \mathrm{S}$ cluster biogenesis rather than directly to $\mathrm{Fe}_{\text {cyt. }}$. Further studies have demonstrated that $\mathrm{Aft} 1 / 2$ regulation depends on core $\mathrm{Fe} / \mathrm{S}$ cluster machinery, but does not require the late-acting factors implicated in the synthesis of [4Fe-4S] clusters (Rutherford et al. 2005). It has been proposed that tripeptide glutathione (GSH) plays an essential role in cellular iron metabolism, whereas its function in thiol redox control is secondary (Kumar et al. 2011). In fact cells with defects in glutathione biosynthesis accumulate iron in mitochondria, display defects in $\mathrm{Fe} / \mathrm{S}$ cluster export from mitochondria and activate the iron regulon (Rutherford et al. 2005; Sipos et al. 2002). Since Fe/S cluster biosynthesis utilizes cytosolic iron as a substrate, this regulatory mechanism links the mitochondrial iron metabolism to cellular iron uptake. The relevance of mitochondrial Fe/S cluster assembly in cellular iron homeostasis is a conserved feature in many eukaryotes. For instance, mammalian cells regulate gene expression according to iron bioavailability through the $\mathrm{Fe} / \mathrm{S}$ cluster status of cytosolic aconitase-related iron regulatory protein IRP1 (reviewed in Muckenthaler et al. 2008).

Mitochondrial exporter Atm1 is essential for inhibiting the Aft1 and Aft2 function, whereas the CIA machinery does not participate in Aft1/2 iron sensing (Hausmann et al. 2008; Rutherford et al. 2005; Ueta et al. 2012). Yeast Atm1 
(ABCB7 in humans) is a conserved protein that exports a sulfur-containing compound produced by the core mitochondrial Fe/S cluster biogenesis system, which is necessary for the maturation of extra-mitochondrial $\mathrm{Fe} / \mathrm{S}$ proteins and to communicate mitochondrial iron demands to Aft1/2 iron regulatory factors (Hausmann et al. 2008). However, it is not known whether the same molecule mediates both processes since the complete nature of this compound still remains elusive. Several hypotheses on the nature of this molecule are currently being considered. It is thought that this compound, denoted X-S, contains sulfur because sulfur donor Nfs1 is required for the process (Kispal et al. 1999; Muhlenhoff et al. 2004). However, whether iron either forms part of the exported moiety to form an $\mathrm{Fe} / \mathrm{S}$ cluster precursor or is incorporated into the cytosol is still to be deciphered. The elucidation of the crystal structure of yeast Atm 1 and a bacterial ABC transporter homolog has recently shown that glutathione associates with the Atm 1 substrate binding cavity, which suggests that glutathione itself or a derivative may form part of the exported entity (Lee et al. 2014; Srinivasan et al. 2014). Transport experiments in inverted membrane vesicles have also shown that Atm1 can transport glutathione disulfide (oxidized GSH or GSSG) and glutathione trisulfide (GS-S-SG, which contains glutathione and an activated persulfide $\mathrm{S}^{0}$ ) in an ATPdependent manner (Schaedler et al. 2014). However, it is not obvious what is the physiological sense of consuming ATP to extract these glutathione derivatives from mitochondria because they can be synthesized in the cytosol (Lill et al. 2014). Various spectroscopic studies have suggested that a $[2 \mathrm{Fe}-2 \mathrm{~S}]^{2+}$ cluster coordinated to four glutathione molecules, $[2 \mathrm{Fe}-2 \mathrm{~S}](\mathrm{GS}) 4^{2-}$ could be a plausible physiological substrate of Atm1-type proteins. First, this glutathione coordinated Fe/S cluster is quite stable under physiological conditions; second, the $[2 \mathrm{Fe}-2 \mathrm{~S}]^{2+}$ core can 
undergo a reversible exchange between the human apo-ISU Fe/S assembly protein and free glutathione; third, the human ISU protein stimulates the formation of $[2 \mathrm{Fe}-2 \mathrm{~S}](\mathrm{GS})_{4}{ }^{2-}$ from iron and sulfide ions in the presence of glutathione; fourth, flow cytometry and photometric iron assays have demonstrated that Atm1 transports the coordinated cluster into proteoliposomes (Li and Cowan 2015; Li et al. 2015; Qi et al. 2012). Consistently with this hypothesis, other ABC transporters, including a bacterial Atm1 homolog, transport heavy metals chelated to glutathione or derivatives (Lee et al. 2014). Nonetheless, the exact identity of X-S and the presence of glutathione or iron in this compound still remain to be elucidated. Multidisciplinary studies will be fundamental to shortly answer this key question.

Once inside the cytoplasm, the mitochondrial exported sulfur-containing molecule is transformed into a $[2 \mathrm{Fe}-2 \mathrm{~S}]^{2+}$ cluster that is inserted into two monothiol glutaredoxins, termed Grx3 and Grx4 (Grx3/4), in a CIA-independent manner (Figure 1) (Muhlenhoff et al. 2010). Multiple genetic and biochemical data have demonstrated the central role played by glutaredoxins Grx3/4 in iron distribution and sensing (Kumanovics et al. 2008; Muhlenhoff et al. 2010; Ojeda et al. 2006; Pujol-Carrion et al. 2006). Both glutaredoxins assemble as homodimers bridged by the $[2 \mathrm{Fe}-2 \mathrm{~S}]^{2+}$ cluster, which is also coordinated to two glutathione molecules (FIgure 1) (Muhlenhoff et al. 2010; Ueta et al. 2012). Grx3/4 belong to the multidomain family of monothiol glutaredoxins (the third one in yeast being mitochondrial Grx5) composed of an amino-terminal thioredoxin (TRX)-like domain and a carboxy-terminal glutaredoxin domain that provides the CGFS active site cysteines, which coordinate the $[2 \mathrm{Fe}-2 \mathrm{~S}]^{2+}$ cluster (Li et al. 2009; Li and Outten 2012). Grx3/4 are homologous, but not totally redundant, as the deletion of either 
gene produces mitochondrial iron accumulation (Pujol-Carrion et al. 2006). The $\mathrm{Fe} / \mathrm{S}$ cluster in the [2Fe-2S] Grx3/4 complex can be used for the biogenesis of cytosolic and nuclear Fe/S proteins, and to communicate mitochondrial iron status to Aft1/2 transcription factors. In an initial iron trafficking step, the [2Fe-2S]bridged Grx3/4 complex, the sulfur-containing intermediate exported from mitochondria and the Tah18-Dre2 electron transport chain contribute to the assembly of a [4Fe-4S] cluster on the Cfd1-Nbp35 scaffold complex in the cytoplasm (reviewed in Lill et al. 2014). Whether Grx3/4 glutaredoxins transfer their $[2 \mathrm{Fe}-2 \mathrm{~S}]^{2+}$ cluster to $\mathrm{Cdf} 1-\mathrm{Nbp} 35$ to assemble the $[4 \mathrm{Fe}-4 \mathrm{~S}]$ center has not been ruled out. Then other later-acting CIA targeting factors transfer the [4Fe-4S] cluster to specific extra-mitochondrial apoproteins. Regarding iron sensing, a combination of biochemical, biophysical and genetic data indicate that [2Fe-2S]bridged Grx3/4 homodimers interact with the BolA-like protein apo-Fra2 and lead to the formation of a [2Fe-2S]Fra2-Grx3/4 complex in which Fra2 substitutes one Grx3/4 monomer and one glutathione molecule (Figure 1) (Kumanovics et al. 2008; Li et al. 2009). In vitro data have demonstrated that this is a thermodynamically and kinetically favorable process (Li et al. 2011a). Further data have indicated that, under iron-sufficient conditions, the [2Fe-2S] cluster is transferred from the Fra2-Grx3/4 complex to Aft1/2 transcription factors. As a result, Aft1/2 factors form [2Fe-2S]-bridged homodimers with decreased affinity for DNA that dissociate from their target promoters and transcription of the iron regulon decreases (Figure 1, dotted lines) (Poor et al. 2014; Ueta et al. 2012). Finally, Aft1/2 factors are exported to the cytoplasm via Msn5 exportin (Ueta et al. 2007). It is still not clear whether the interaction between the Grx3/4 complex and Aft1/2 transcription factors occurs in the cytoplasm, in the nucleus, or in both 
(Ojeda et al. 2006; Pujol-Carrion et al. 2006). Some studies have shown that Grx3 is predominantly cytoplasmic and that tethering Grx4 to the mitochondrial outer membrane does not relieve its inhibitory effect on Aft1, which suggests a cytoplasmic interaction. Nonetheless, a nuclear interaction is not ruled out because Grx3/4 can also localize to the nucleus (Kumanovics et al. 2008; Ojeda et al. 2006; Pujol-Carrion et al. 2006). The conserved cysteine residue at the Grx3/4 CGFS active site is required for iron incorporation and the interaction with Aft1 (and presumably Aft2), whereas the conserved CDC motif in Aft1/2 is necessary for their interaction with Grx3/4, Msn5 and the Fe/S cluster (Ojeda et al. 2006; Poor et al. 2014; Pujol-Carrion et al. 2006; Ueta et al. 2007; Ueta et al. 2012). Iron deficiency releases Aft1/2, which accumulates into the nucleus, binds to FeREs and activates the transcription of iron regulon genes (Figure 1, red continuous lines). Activation of the iron regulon is also observed in mutants at the conserved Aft1/2 $\mathrm{CDC}$ motif (Aft1/2 ${ }^{\mathrm{UP}}$ mutants) and in core mitochondrial Fe/S cluster synthesis and export mutants (Rutherford et al. 2005; Ueta et al. 2007; Yamaguchi-Iwai et al. 1995).

The role of Grx3/4 and Fra2 in iron sensing or regulation is conserved in many organisms, including other fungi and humans. In $S$ pombe, Grx4 forms a heterodimer with Fra2, which participates in the regulation of Fep4, the repressor of iron acquisition genes, to which it seems to bind in the nucleus (Jacques et al. 2014). At the same time, Grx4 interacts in an iron-dependent and ironindependent manner with $\mathrm{Php} 4$, a repressor of iron-dependent genes, whereas Fra2 might play a partial role in the process (Encinar del Dedo et al. 2015; Labbe et al. 2013). In humans, GRX3, which contains one TRX domain and two GRX domains, forms an heterotrimer with two BOLA2 molecules (the yeast Fra2 homolog) 
bridged by two $[2 \mathrm{Fe}-2 \mathrm{~S}]^{2+}$ clusters, and participates in iron trafficking by transferring both clusters to apo-anamorsin (the yeast Dre2 homolog) (Banci et al. 2015; Li et al. 2012a). In S. cerevisiae, an aminopeptidase P-like protein termed Fra1, participates in the iron sensing pathway by interacting with Fra2 and Grx3/4 (Kumanovics et al. 2008). To our knowledge, no iron-related role has been described for the S. pombe $\left(f r a 1^{+}\right)$and human (XNPEP1) homologs.

Various observations suggest that in addition to the Fe/S signaling pathway, there may be a second mechanism that senses $\mathrm{Fe}_{\text {cyt }}$ and regulates the Aft1 function. First, the iron regulon is not completely active in fra1 $\Delta$ and fra $2 \Delta$ mutants, nor in strains defective in mitochondrial Fe/S cluster biogenesis (Kumanovics et al. 2008). Second, iron depletion further augments Aft1 transcriptional activity in

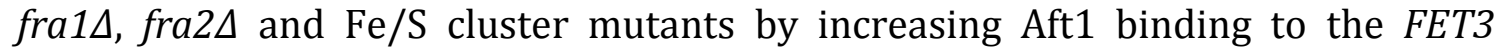
promoter (Kumanovics et al. 2008). Third, the iron regulon is induced when mitochondrial iron importers $M R S 3$ and MRS4 are either deleted, which leads to reduced $\mathrm{Fe} / \mathrm{S}$ synthesis, or overexpressed, which would cause cytosolic iron depletion while the Fe/S cluster system would be active (Muhlenhoff et al. 2003). Fourth, the mathematical modeling of yeast iron regulation also supports the implication of $\mathrm{Fe}_{\text {cyt }}$ as a regulatory sensor (Wofford and Lindahl 2015). These authors consider the possibility that $\mathrm{Fe}_{\text {cyt }}$ reacts in the cytoplasm with the sulfurcontaining compound exported from mitochondria to generate the Fe-S cluster, which binds to the Grx3/4 homodimer. Thus the dominant role in iron sensing would be played by the limiting factor, either Fe $\mathrm{e}_{\text {cyt }}$ or X-S (Wofford and Lindahl 2015). Therefore, a dual sensing regulation based on both mitochondrial $\mathrm{Fe} / \mathrm{S}$ cluster biogenesis and $\mathrm{Fe}_{\text {cyt }}$ seems to control Aft1/2 in response to alterations in 
iron bioavailability. In any case, further work is necessary to fully decipher the detailed sensing and regulatory mechanisms that control yeast iron homeostasis.

Finally, it needs be remarked that, while a common way to measure the iron regulon expression consists in fusions of the FET3 promoter to the LacZ reporter, not all genes in the regulon are equally regulated by iron; e.g., under heme depletion, FET3 is repressed independently of iron levels because Aft1, which is bound to its promoter under low iron conditions, interacts with repressor Tup1 and histone deacetylase Hda1, while other genes of the regulon, like ARN1 or FIT1, recruit Cti6 to overcome this repression (Crisp et al. 2006).

\section{Regulation of Yap5 in response to Fe excess.}

Yeast cells utilize various mechanisms to protect themselves from the deleterious effects of iron overload. Transcription factor Yap5 coordinates key responses to elevated iron levels by activating the expression of a few target genes upon iron overload. The first identified Yap5-target was CCC1, which encodes a transporter that mediates the import of excess cytosolic iron into the vacuole, the major iron storage and detoxifying organelle in yeast (Li et al. 2008). The relevance of the Ccc1 function under excess iron is highlighted by the extreme sensitivity of ccc1 1 mutants to high iron concentrations (Li et al. 2001). However, Yap5 is not the only transcription factor to regulate $C C C 1$ expression since its mRNA levels are still up-regulated by iron in yap5 5 mutant cells by an unknown factor (Pimentel et al. 2012). Consistently with this, $\operatorname{ccc} 1 \Delta$ strains are more sensitive to excess iron than yap5 5 mutants (Pimentel et al. 2012). Yap5 also activates the expression of three additional genes in response to elevated iron concentrations: GRX4, TYW1 and CUP1 (Table 2; Li et al. 2011b; Pimentel et al. 2012). As described above, Grx4 
monothiol glutaredoxin participates in the transmission of the mitochondrial iron signal that inactivates Aft1/2 transcriptional function upon iron sufficiency. Therefore, activation of GRX4 transcription by Yap5 has been proposed to contribute to inhibit the Aft1/2 function when iron levels rise. Accordingly, yap5 $\Delta$ mutants display altered Aft1 nuclear localization and gene target expression patterns (Pimentel et al. 2012). Furthermore, TYW1 encodes a [4Fe-4S]-containing enzyme required for the biosynthesis of wybutosine-modified tRNA. Several lines of evidence have suggested that Tyw1 protects cells from iron toxicity by sequestering cytosolic free iron as protein-bound Fe/S clusters, but not through its nucleoside biosynthesis function (Li et al. 2011b). Finally, Yap5 activates the expression of cytosolic copper-binding metallothionein Cup1 to probably limit copper availability to Fet3, the multicopper ferroxidase component of the reductive high affinity iron uptake system.

Yap5 belongs to a fungi-specific family of basic leucine zipper (bZIP) transcription regulators, composed of yeast of eight members, including the major oxidative stress regulator Yap1 (Rodrigues-Pousada et al. 2010). Unlike Aft1, the Yap5 protein localizes to the nucleus independently of cellular iron levels (Li et al. 2008). Yap5 is composed of an amino-terminal bZIP DNA-binding domain and a carboxy-terminal activation domain. The DNA-binding domain allows constitutive Yap5 association with TTA(C/G)TAA consensus sequences within the promoter of its target genes (Li et al. 2008). Elegant genetic and biochemical studies have demonstrated that, similarly to Aft $1 / 2$ transcription factors, the Yap5 protein does not sense cytosolic iron levels, but is regulated instead by the $\mathrm{Fe} / \mathrm{S}$ clusters synthesized in mitochondria (Figure 1) (Li et al. 2012b). Accordingly, Yap5 activation is prevented in cells defective in mitochondrial $\mathrm{Fe} / \mathrm{S}$ biogenesis (Li et al. 
2012b). However, neither cytosolic Fe/S cluster machinery nor monothiol glutaredoxins Grx3/4 are required for Yap5 transcriptional activation upon iron overload (Figure 1) (Li et al. 2012b). Moreover, Yap5 regulation by iron occurs properly under anaerobic conditions and in cells with increased oxidants, which indicates that Yap5 specifically responds to Fe/S clusters rather than to oxidative stresses (Li et al. 2012b). The Yap5 activator region contains two nearby cysteinerich domains, known as n-CRD and c-CRD, that regulate its activity depending on iron availability. The mutagenesis of any of the four conserved cysteine residues within n-CRD strongly limits Yap5 iron-dependent transcription induction and causes iron sensitivity (Li et al. 2008). Further mutagenesis of the three conserved cysteine residues in c-CRD further exacerbates the Yap5 defect in iron-mediated transcriptional activation ( $\mathrm{Li}$ et al. 2008). Therefore, iron promotes Yap5 transcriptional activity through conserved cysteine residues in CRDs, but does not affect Yap5 DNA binding capacity. Recent biochemical and biophysical data have also demonstrated that a [2Fe-2S] cluster can stably bind to Yap5 n-CRD in vivo, whereas c-CRD probably associates with a second [2Fe-2S] cluster in a more labile fashion (Rietzschel et al. 2015). Despite their divergent Fe/S cluster-binding affinity, both CRDs significantly contribute to the Yap5 regulatory function (Rietzschel et al. 2015). In vitro studies have also demonstrated that Fe/S clusterbinding to any of the CRDs induces a conformational change in the Yap5 activation domain, which probably triggers transcriptional activation (Rietzschel et al. 2015). Importantly, this Fe/S-binding motif seems conserved since transcription factor HapX from pathogenic fungi, such as Aspergillus fumigatus, utilize an n-CRD homologous to that of Yap5 to activate CCC1 expression upon iron excess (Gsaller et al. 2014). 


\section{Divergence of iron metabolism in yeast strains from different origins}

Iron homeostasis has been profoundly studied in laboratory $S$. cerevisiae strains, but very little is known about how yeast strains from different geographical origins and sources respond to alterations in iron bioavailability. Over the last decade, the number of complete $S$. cerevisiae genome sequences has dramatically increased, and now includes domesticated strains from different fermentations (wine, beer, bread, sake, etc), dietetic and clinical strains, and isolates from diverse natural environments (Liti et al. 2009). Such vast genomic information is facilitating the study of the mechanisms that yeasts have developed to adapt to different environments. Comparative studies have uncovered major divergences in iron metabolism among different yeast strains (Lee et al. 2013; Martinez-Garay et al. 2016). A phenotypic analysis of a large number of $S$. cerevisiae strains has shown that strains resistant to elevated iron concentrations limit iron acquisition and are sensitive to low iron conditions, whereas strains sensitive to excess iron display increased iron uptake and are better adapted to iron deficiency (Table 3; Martinez-Garay et al. 2016). Although the molecular bases of this behavior have not been elucidated, preliminary data suggest alterations in iron sensing and regulation (Martinez-Garay et al. 2016). For instance, an expression profile analysis has shown that, relative to other strains, wild Malaysian yeast isolates from palm flowers repress iron uptake genes and induce iron toxicity responses when grown under standard laboratory conditions (Lee et al. 2013). Reciprocal hemizygote analyses have suggested that this phenotype is attributable to a partial loss-of-function of iron detoxifying vacuolar transporter 
Ccc1 (Lee et al. 2013), which might lead to larger cytosolic and/or mitochondrial iron pools that alter the expression of iron-related genes (Table 3). The hemizygote expression of the Malaysian AFT1 ortholog up-regulates the iron regulon, whereas the Malaysian allele of YAP5 reduces the expression of iron resistance genes (Lee et al. 2013). These results are consistent with a gain-offunction of the Malaysian AFT1 allele and a loss-of-function of the YAP5 allele, which are not manifested in the parental strain, probably due to the dominant effect exerted by the defective Ccc1 transporter (Table 3). Therefore, the Malaysian population has diverged toward specialization in iron deficiency due to the selective pressure of the environment, and has weakened its resistance to excess iron. In fact, these Malaysian strains grow efficiently under low iron conditions, but are sensitive to excess iron (Lee et al. 2013; Martinez-Garay et al. 2016). Further studies with different yeast strains could significantly contribute to our current understanding of iron sensing and regulation.

\section{Acknowledgements}

We apologize to those whose work has not been cited due to space limitations. We are grateful to Anto Romero and Lucía Ramos-Alonso for critically reading this manuscript. Ana Perea-García is a recipient of a postdoctoral "Juan de la Cierva-Formación" contract from the Spanish Ministry of Economy and Competitiveness. Work in our group is supported by the BI02014-56298-P grant from the Spanish Ministry of Economy and Competitiveness, and the AICO/2015/004 grant from the "Generalitat Valenciana" to Sergi Puig and María Teresa Martínez-Pastor, respectively. 


\section{Figure legends}

Figure 1. Proposed model for the regulation of yeast iron-responsive transcription factors Aft1/2 and Yap5 by $\mathrm{Fe} / \mathrm{S}$ cluster biosynthesis. Dashed lines show the situation upon iron excess $(+\mathrm{Fe})$, whereas red lines refer to iron deficiency (-Fe). 


\section{References}

An X, Zhang C, Sclafani RA, Seligman P, Huang M (2015) The late-annotated small ORF LSO1 is a target gene of the iron regulon of Saccharomyces cerevisiae. Microbiol Open 4:941-951. doi: 10.1002/mbo3.303

Banci L, Ciofi-Baffoni S, Gajda K, Muzzioli R, Peruzzini R, Winkelmann J (2015) Nterminal domains mediate [2Fe-2S] cluster transfer from glutaredoxin-3 to anamorsin. Nat Chem Biol 11:772-778 doi:10.1038/nchembio.1892

Blaiseau PL, Lesuisse E, Camadro JM (2001) Aft2p, a novel iron-regulated transcription activator that modulates, with Aft1p, intracellular iron use and resistance to oxidative stress in yeast. J Biol Chem 276:34221-34226.

Chen OS, Crisp RJ, Valachovic M, Bard M, Winge DR, Kaplan J (2004) Transcription of the yeast iron regulon does not respond directly to iron but rather to iron-sulfur cluster biosynthesis. J Biol Chem 279:29513-29518

Chen OS, Hemenway S, Kaplan J (2002) Inhibition of Fe-S cluster biosynthesis decreases mitochondrial iron export: evidence that Yfh1p affects Fe-S cluster synthesis Proc Natl Acad Sci USA. 99:12321-12326 doi:10.1073/pnas.192449599

Courel M, Lallet S, Camadro JM, Blaiseau PL (2005) Direct activation of genes involved in intracellular iron use by the yeast iron-responsive transcriptin factor Aft2 without its paralog Aft1. Mol Cell Biol 25:6760-6771

Crisp RJ, Adkins EM, Kimmel E, Kaplan J (2006) Recruitment of Tup1p and Cti6p regulates heme-deficient expression of Aft1p target genes. EMBO J 25:512521 doi:10.1038/sj.emboj.7600961

Encinar del Dedo J, Gabrielli N, Carmona M, Ayte J, Hidalgo E (2015) A cascade of iron-containing proteins governs the genetic iron starvation response to 
promote iron uptake and inhibit iron storage in fission yeast. PLoS Genet 11:e1005106 doi:10.1371/journal.pgen.1005106

Gsaller F et al. (2014) The Janus transcription factor HapX controls fungal adaptation to both iron starvation and iron excess. EMBO J 33:2261-2276 doi:10.15252/embj.201489468

Hausmann A, Samans B, Lill R, Muhlenhoff U (2008) Cellular and mitochondrial remodeling upon defects in iron-sulfur protein biogenesis. J Biol Chem 283:8318-8330

Jacques JF, Mercier A, Brault A, Mourer T, Labbe S (2014) Fra2 is a co-regulator of Fep1 inhibition in response to iron starvation. PLoS One 9:e98959 doi:10.1371/journal.pone.0098959

Kaplan CD, Kaplan J (2009) Iron acquisition and transcriptional regulation. Chem $\operatorname{Rev} 109: 4536-4552$

Kispal G, Csere P, Prohl C, Lill R (1999) The mitochondrial proteins Atm1p and Nfs1p are essential for biogenesis of cytosolic Fe/S proteins. EMBO J 18:3981-3989 doi:10.1093/emboj/18.14.3981

Kumanovics A et al. (2008) Identification of FRA1 and FRA2 as genes involved in regulating the yeast iron regulon in response to decreased mitochondrial iron-sulfur cluster synthesis. J Biol Chem 283:10276-10286

Kumar C et al. (2011) Glutathione revisited: a vital function in iron metabolism and ancillary role in thiol-redox control. EMBO J 30:2044-2056 doi:10.1038/emboj.2011.105

Labbe S, Khan MG, Jacques JF (2013) Iron uptake and regulation in Schizosaccharomyces pombe. Curr Opin Microbiol 16:669-676 doi:10.1016/j.mib.2013.07.007 
Lee HN, Mostovoy Y, Hsu TY, Chang AH, Brem RB (2013) Divergence of iron metabolism in wild Malaysian yeast. G3 (Bethesda) 3:2187-2194 doi:10.1534/g3.113.008011

Lee JY, Yang JG, Zhitnitsky D, Lewinson O, Rees DC (2014) Structural basis for heavy metal detoxification by an Atm1-type ABC exporter. Science 343:1133-1136 doi:10.1126/science.1246489

Li H et al. (2011a) Histidine 103 in Fra2 is an iron-sulfur cluster ligand in the [2Fe2S] Fra2-Grx3 complex and is required for in vivo iron signaling in yeast. J Biol Chem 286:867-876 doi:10.1074/jbc.M110.184176

Li H et al. (2009) The yeast iron regulatory proteins Grx3/4 and Fra2 form heterodimeric complexes containing a [2Fe-2S] cluster with cysteinyl and histidyl ligation. Biochemistry 48:9569-9581 doi:10.1021/bi901182w

Li H, Mapolelo DT, Randeniya S, Johnson MK, Outten CE (2012a) Human glutaredoxin 3 forms [2Fe-2S]-bridged complexes with human BolA2. Biochemistry 51:1687-1696 doi:10.1021/bi2019089

Li H, Outten CE (2012) Monothiol CGFS glutaredoxins and BolA-like proteins: [2Fe2S] binding partners in iron homeostasis. Biochemistry 51:4377-4389 doi:10.1021/bi300393z

Li J, Cowan JA (2015) Glutathione-coordinated [2Fe-2S] cluster: a viable physiological substrate for mitochondrial ABCB7 transport. Chem Commun (Camb) 51:2253-2255 doi:10.1039/c4cc09175b

Li J, Pearson SA, Fenk KD, Cowan JA (2015) Glutathione-coordinated [2Fe-2S] cluster is stabilized by intramolecular salt bridges. J Biol Inorg Chem 20:1221-1227 doi:10.1007/s00775-015-1301-3 
Li L, Bagley D, Ward DM, Kaplan J (2008) Yap5 is an iron-responsive transcriptional activator that regulates vacuolar iron storage in yeast. Mol Cell Biol 28:1326-1337

Li L, Chen OS, McVey Ward D, Kaplan J (2001) CCC1 is a transporter that mediates vacuolar iron storage in yeast. J Biol Chem 276:29515-29519

Li L, Jia X, Ward DM, Kaplan J (2011b) Yap5 protein-regulated transcription of the TYW1 gene protects yeast from high iron toxicity. J Biol Chem 286:3848838497 doi:10.1074/jbc.M111.286666

Li L, Miao R, Bertram S, Jia X, Ward DM, Kaplan J (2012b) A role for iron-sulfur clusters in the regulation of transcription factor Yap5-dependent high iron transcriptional responses in yeast. J Biol Chem 287:35709-35721 doi:10.1074/jbc.M112.395533

Lill R, Srinivasan V, Muhlenhoff U (2014) The role of mitochondria in cytosolicnuclear iron-sulfur protein biogenesis and in cellular iron regulation. Curr Opin Microbiol 22:111-119 doi:10.1016/j.mib.2014.09.015

Liti G et al. (2009) Population genomics of domestic and wild yeasts. Nature 458:337-341 doi:10.1038/nature07743

Martinez-Garay CA, de Llanos R, Romero AM, Martinez-Pastor MT, Puig S (2016) Responses of Saccharomyces cerevisiae Strains from Different Origins to Elevated Iron Concentrations. Appl Environ Microbiol 82:1906-1916 doi:10.1128/AEM.03464-15

Muckenthaler MU, Galy B, Hentze MW (2008) Systemic iron homeostasis and the iron-responsive element/iron-regulatory protein (IRE/IRP) regulatory network. Annu Rev Nutr 28:197-213 
Muhlenhoff U, Balk J, Richhardt N, Kaiser JT, Sipos K, Kispal G, Lill R (2004) Functional characterization of the eukaryotic cysteine desulfurase Nfs1p from Saccharomyces cerevisiae. J Biol Chem 279:36906-36915 doi:10.1074/jbc.M406516200

Muhlenhoff U et al. (2015) Compartmentalization of iron between mitochondria and the cytosol and its regulation. Eur J Cell Biol 94:292-308 doi:10.1016/j.ejcb.2015.05.003

Muhlenhoff $U$ et al. (2010) Cytosolic monothiol glutaredoxins function in intracellular iron sensing and trafficking via their bound iron-sulfur cluster. Cell Metab 12:373-385

Muhlenhoff $U$ et al. (2003) A specific role of the yeast mitochondrial carriers Mrs3/4p in mitochondrial iron acquisition under iron-limiting conditions. J Biol Chem 278:40612-40620

Ojeda L, Keller G, Muhlenhoff U, Rutherford JC, Lill R, Winge DR (2006) Role of glutaredoxin-3 and glutaredoxin-4 in the iron regulation of the Aft1 transcriptional activator in Saccharomyces cerevisiae. J Biol Chem 281:17661-17669

Outten CE, Albetel AN (2013) Iron sensing and regulation in Saccharomyces cerevisiae: Ironing out the mechanistic details. Curr Opin Microbiol 16:662668 doi:10.1016/j.mib.2013.07.020

Philpott CC, Leidgens S, Frey AG (2012) Metabolic remodeling in iron-deficient fungi. Biochim $\quad$ Biophys $\quad$ Acta $1823: 1509-1520$ doi:10.1016/j.bbamcr.2012.01.012

Pimentel C, Vicente C, Menezes RA, Caetano S, Carreto L, Rodrigues-Pousada C (2012) The role of the Yap5 transcription factor in remodeling gene 
expression in response to $\mathrm{Fe}$ bioavailability. PLoS One 7:e37434 doi:10.1371/journal.pone.0037434

Poor CB et al. (2014) Molecular mechanism and structure of the Saccharomyces cerevisiae iron regulator Aft2. Proc Natl Acad Sci USA 111:4043-4048 doi:10.1073/pnas.1318869111

Puig S, Askeland E, Thiele DJ (2005) Coordinated remodeling of cellular metabolism during iron deficiency through targeted mRNA degradation. Cell 120:99-110. doi 10.1016/j.cell.2004.11.032

Puig S, Vergara SV, Thiele DJ (2008) Cooperation of two mRNA-binding proteins drives metabolic adaptation to iron deficiency. Cell Metab 7:555-564. doi 10.1016/j.cmet.2008.04.010

Pujol-Carrion N, Belli G, Herrero E, Nogues A, de la Torre-Ruiz MA (2006) Glutaredoxins Grx3 and Grx4 regulate nuclear localisation of Aft1 and the oxidative stress response in Saccharomyces cerevisiae. J Cell Sci 119:45544564

Qi W, Li J, Chain CY, Pasquevich GA, Pasquevich AF, Cowan JA (2012) Glutathione complexed Fe-S centers. J Am Chem Soc 134:10745-10748 doi:10.1021/ja302186j

Rietzschel N, Pierik AJ, Bill E, Lill R, Muhlenhoff U (2015) The basic leucine zipper stress response regulator Yap5 senses high-iron conditions by coordination of [2Fe-2S] clusters. Mol Cell Biol 35:370-378 doi:10.1128/MCB.01033-14

Rodrigues-Pousada C, Menezes RA, Pimentel C (2010) The Yap family and its role in stress response. Yeast 27:245-258 doi:10.1002/yea.1752 
Rutherford JC, Jaron S, Ray E, Brown PO, Winge DR (2001) A second ironregulatory system in yeast independent of Aft1p. Proc Natl Acad Sci USA 98:14322-14327

Rutherford JC, Jaron S, Winge DR (2003) Aft1p and Aft2p mediate iron-responsive gene expression in yeast through related promoter elements. J Biol Chem $278: 27636-27643$

Rutherford JC, Ojeda L, Balk J, Muhlenhoff U, Lill R, Winge DR (2005) Activation of the iron regulon by the yeast Aft1/Aft2 transcription factors depends on mitochondrial but not cytosolic iron-sulfur protein biogenesis. J Biol Chem 280:10135-10140

Sanvisens N, Puig S (2011) Causes and consequences of nutritional iron deficiency in living organisms. In: Merkin TC (ed) Biology of starvation in humans and other organisms. Nova Science Publishers, pp 245-276

Schaedler TA, Thornton JD, Kruse I, Schwarzlander M, Meyer AJ, van Veen HW, Balk J (2014) A conserved mitochondrial ATP-binding cassette transporter exports glutathione polysulfide for cytosolic metal cofactor assembly. J Biol Chem 289:23264-23274 doi:10.1074/jbc.M114.553438

Shakoury-Elizeh M et al. (2004) Transcriptional remodeling in response to iron deficiency in Saccharomyces cerevisiae. Mol Biol Cell 15:1233-1243

Sipos K, Lange H, Fekete Z, Ullmann P, Lill R, Kispal G (2002) Maturation of cytosolic iron-sulfur proteins requires glutathione. J Biol Chem 277:2694426949 doi:10.1074/jbc.M200677200

Srinivasan V, Pierik AJ, Lill R (2014) Crystal structures of nucleotide-free and glutathione-bound mitochondrial ABC transporter Atm1. Science 343:11371140 doi:10.1126/science.1246729 
Ueta R, Fujiwara N, Iwai K, Yamaguchi-Iwai Y (2007) Mechanism underlying the iron-dependent nuclear export of the iron-responsive transcription factor Aft1p in Saccharomyces cerevisiae. Mol Biol Cell 18:2980-2990

Ueta R, Fujiwara N, Iwai K, Yamaguchi-Iwai Y (2012) Iron-induced dissociation of the Aft1p transcriptional regulator from target gene promoters is an initial event in iron-dependent gene suppression. Mol Cell Biol 32:4998-5008

Wofford JD, Lindahl PA (2015) Mitochondrial Iron-Sulfur Cluster Activity and Cytosolic Iron Regulate Iron Traffic in Saccharomyces cerevisiae. J Biol Chem 290:26968-26977 doi:10.1074/jbc.M115.676668

Waters BM, Eide DJ (2002) Combinatorial control of yeast FET4 gene expression by iron, zinc, and oxygen. J Biol Chem 277:33749-33757

Wu X et al. (2016) Potassium and the K/H exchanger Kha1p promote binding of copper to apo-Fet3 multi-copper ferroxidase. J Biol Chem 291:9796-9806. doi: $10.1074 / j b c . M 115.700500$

Yamaguchi-Iwai Y, Dancis A, Klausner RD (1995) AFT1: a mediator of iron regulated transcriptional control in Saccharomyces cerevisiae. EMBO J $14: 1231-1239$

Yamaguchi-Iwai Y, Ueta R, Fukunaka A, Sasaki R (2002) Subcellular localization of Aft1 transcription factor responds to iron status in Saccharomyces cerevisiae. J Biol Chem 277:18914-18918 


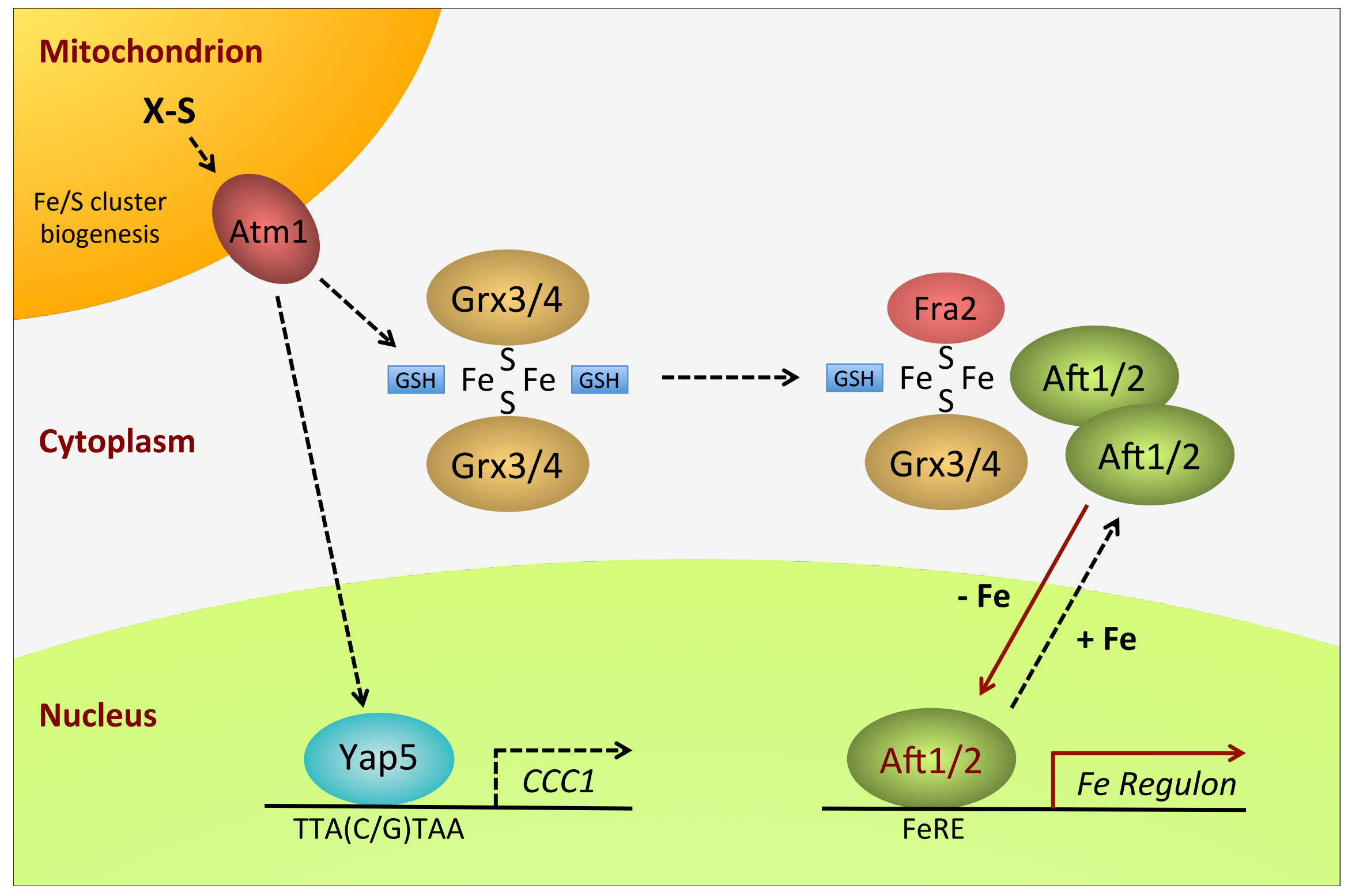


Table 1. Genes transcriptionally activated by Aft1 and Aft2 in response to iron deficiency. Data collected from An et al. 2015; Courel et al. 2005; Puig et al. 2005; Puig et al. 2008; Rutherford et al. 2001; Rutherford et al. 2003; ShakouryElizeh et al. 2004; Waters and Eide 2002; and Wu et al. 2016.

\begin{tabular}{|c|c|c|}
\hline Name & Function & Localization \\
\hline FRE1 & Metalloreductase & Plasma membrane \\
\hline FRE2 & Metalloreductase & Plasma membrane \\
\hline FRE3 & Metalloreductase & Plasma membrane \\
\hline FRE4 & Metalloreductase & Plasma membrane \\
\hline FRE5 & Metalloreductase & Mitochondria \\
\hline FRE6 & Metalloreductase & Vacuolar membrane \\
\hline FET3 & High-affinity iron transport & Plasma membrane \\
\hline FTR1 & High-affinity iron transport & Plasma membrane \\
\hline$A T X 1$ & Copper chaperone & Cytoplasm \\
\hline CCC 2 & Copper-transporting ATPase & Trans-Golgi network \\
\hline FET4 & Low-affinity iron transport & Plasma membrane \\
\hline ARN1 & Iron siderophore transport & Plasma membrane \\
\hline ARN2/TAF1 & Iron siderophore transport & Plasma membrane \\
\hline ARN3/SIT1 & Iron siderophore transport & Plasma membrane \\
\hline$A R N 4 / E N B 1$ & Iron siderophore transport & Plasma membrane \\
\hline FIT1 & Iron siderophore transport & Cell wall \\
\hline FIT2 & Iron siderophore transport & Cell wall \\
\hline FIT3 & Iron siderophore transport & Cell wall \\
\hline FET5 & High-affinity iron transport & Vacuolar membrane \\
\hline FTH1 & High-affinity iron transport & Vacuolar membrane \\
\hline
\end{tabular}




$\begin{array}{lll}\text { SMF3 } & \text { Low-affinity iron transport } & \text { Vacuolar membrane } \\ \text { MRS4 } & \text { Mitochondrial iron transport } & \text { Mitochondrial inner membrane } \\ \text { ISU1 } & \text { Fe/S cluster synthesis } & \text { Mitochondrial matrix } \\ \text { HMX1 } & \text { Heme oxygenase } & \text { Endoplasmic reticulum } \\ \text { VHT1 } & \text { High-affinity biotin transport } & \text { Plasma membrane } \\ \text { CTH1 } & \text { Post-transcriptional regulation } & \text { Nucleus/Cytoplasm } \\ \text { CTH2/TIS11 } & \text { Post-transcriptional regulation } & \text { Nucleus/Cytoplasm } \\ \text { COT1 } & \text { Zinc transport } & \text { Vacuolar membrane } \\ \text { LSO1 } & \text { Unknown function } & \text { Nucleus/Cytoplasm } \\ \text { KHA1 } & \mathrm{K}^{+} / \mathrm{H}^{+} \text {antiporter } & \text { Trans-Golgi network }\end{array}$


Table 2. Genes transcriptionally activated by Yap5 in response to excess iron.

Data collected from Li et al. 2008; Li et al. 2011b; and Pimentel et al, 2012

$\begin{array}{lll}\text { Name } & \text { Function } & \text { Localization } \\ \text { CCC1 } & \text { Iron transport } & \text { Vacuolar membrane } \\ \text { GRX4 } & \text { Iron sensing } & \text { Nucleus/Cytoplasm } \\ \text { TYW1 } & \text { Iron binding } & \text { Cytoplasm } \\ \text { CUP1 } & \text { Copper binding } & \text { Nucleus/Cytoplasm }\end{array}$


Table 3. Divergent response to iron availability in yeast strains from different origins. The phenotypic analysis of many S. cerevisiae strains grown in media with different iron availability has allowed the identification of two groups of strains that differ from laboratory strains: iron-resistant strains and ironsensitive strains (Lee et al. 2013; Martinez-Garay et al. 2016). Kyokai 6 is a $S$. cerevisiae strain isolated from sake fermentations in Japan. UWOPS03-461.4 is a $S$. cerevisiae strain isolated from palm flowers in Malaysia.

\begin{tabular}{|c|c|c|}
\hline Strains & Iron-resistant & Iron-sensitive \\
\hline Growth in iron excess & Increased & Decreased \\
\hline Iron accumulation & Decreased & Increased \\
\hline Growth in iron deficiency & Decreased & Increased \\
\hline Examples & Kyokai 6 & UWOPS03-461.4 \\
\hline Gain-of-function alleles & Unknown & AFT1 \\
\hline Loss-of-function alleles & Unknown & CCC1, YAP5 \\
\hline
\end{tabular}

